Nanotechnology

\section{Nanoparticles go with the flow}

Adv. Mater. doi:10.1002/adma.200401060 (2004)

In the absence of a solvent, nanoparticles behave like solids. But Athanasios B. Bourlinos et al. have come up with a way of changing nanoparticles into a liquid without using a solvent in which to dissolve or suspend them.

The authors coated silica nanoparticles (seven nanometres in diameter) with chemical groups borrowed from another burgeoning area of chemistry - ionic liquids. These chemical groups, made of long carbon chains with extra silicon and nitrogen atoms, give the nanoparticles a positive charge. By balancing this charge with a suitable negative ion, Bourlinos et al. created a 'nanosalt' whose physical properties could be tailored to make it a liquid at room temperature.

They performed the same trick using iron-oxide nanoparticles, producing a magnetic liquid that they describe as the first example of a solvent-free ferrofluid.

These liquid nanosalts, say Bourlinos et al., are a unique class of solvent-free colloids, which could produce thin films of nanoparticles more easily or make useful solvents for chemical reactions. Mark Peplow

\section{Genetics}

\section{Fragile fertility}

Proc. R. Soc. Lond. B doi:10.1098/rspb.2004.2903 (2004)

When it comes to mating, it seems that males are the sensitive type. Ilik J. Saccheri and colleagues' study of butterfly fertility shows that the sterility effect resulting from inbreeding is largely restricted to males.

Inbreeding generally leads to a decrease in the genetic variability of a population, often with negative effects, because detrimental gene variants are not 'watered down'. Mated siblings of the Bicyclus anynana butterfly species, for instance, often produce eggs that do not hatch.

To investigate further, Saccheri et al. established six inbred lines of B. anynana and conducted various crosses, pairing males and females within and between these lines. By analysing the results of the different crosses, the authors deduced that inbred males were far more often to blame than inbred females for the reduction in egghatching. In other words, the reproductive fitness of males suffers more than that of females as a result of inbreeding.

Why this should happen remains uncertain. As the authors point out, the production of sperm has been suggested to be less developmentally stable than that of eggs, but this is probably not the only mechanism involved.

Roxanne Khams

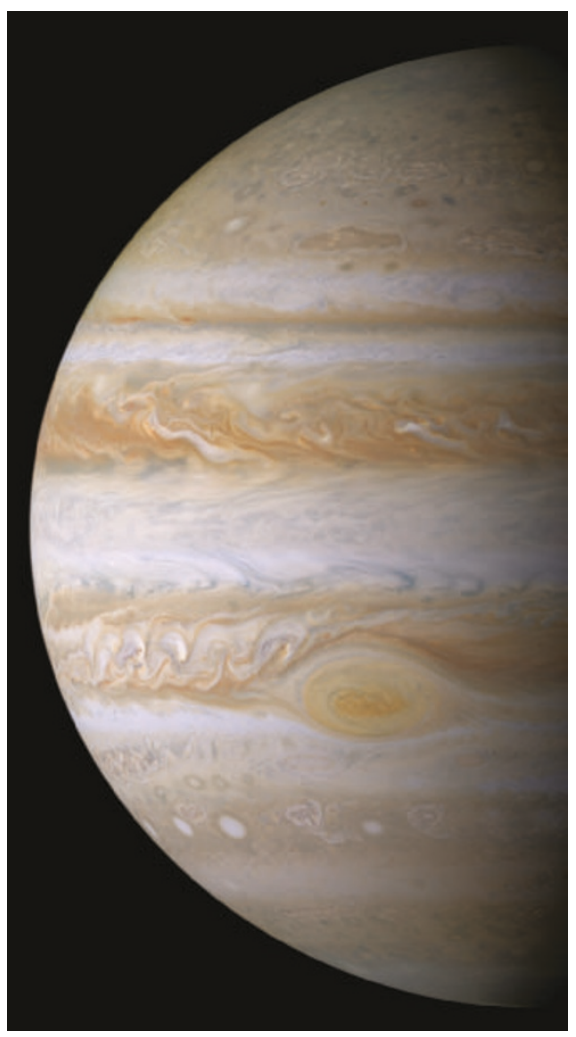

Fluid dynamics

\section{Jove's jet engine}

Geophys. Res. Lett. 31, L22701 (2004)

Jupiter's colourful atmosphere has a characteristic banded pattern: 'zonal jets' flow in alternating directions, parallel to lines of latitude. Saturn's atmosphere has this structure too, and there are even faint zonal jets in Earth's oceans. Yet the origin of the banding is still not clear. The favoured hypothesis is that the jets are coherent structures that organize themselves within a shallow layer of turbulent fluid as a result of the planet's rotation thanks to the so-called 'planetary $\beta$ effect' - the latitudinal variation of the Coriolis force.

Such an origin is supported by numerical calculations, but experiments mimicking a planetary atmosphere are challenging: to make the fluid sufficiently turbulent, the scale of the simulation needs to be very large. P. L. Read et al. have now achieved this by using a turntable $14 \mathrm{~m}$ in diameter, supporting a shallow layer of salty water in which convection (imitating that in the gas-giant atmospheres) is maintained by spraying denser, saltier water on top. The planetary $\beta$ effect is reproduced topographically by a 'conical' slope in the bottom of the fluid tank. Small particles suspended in the flow reveal banded zoning, as small turbulent eddies conspire to generate a large-scale coherent pattern. The jets are not as neat as those on Jupiter, but they show that the basic idea works.
Biological hydration Grease makes ions stickier

Phys. Rev. Lett. 93, 228104 (2004)

Hydrophobic interaction - the apparent attraction between hydrophobic species in water - is considered a key factor in maintaining the correct folded conformation of a protein molecule. This attraction is thought to result, in a way that is still imperfectly understood, from changes in the arrangement of hydrogen bonds between water molecules surrounding a hydrophobe.

Florin Despa et al. have now identified a possible new role for water in protein folding. They calculate that water molecules in the hydration shell of a hydrophobic unit move an order of magnitude more slowly than those in the bulk, and that the dielectric constant of this water layer is significantly reduced.

Water's high dielectric constant is the reason it is a good solvent for ions: it screens their electrical charges, preventing them from aggregating. But in the vicinity of hydrophobic residues in a protein chain, the reduction in dielectric constant strengthens the attraction between charged residues, potentially helping to fix the protein's folds in place. Moreover, the effect means that water confined in nanoscale hydrophobic environments might have quite different solvent properties from those of the bulk liquid.

Philip Ball

\section{Microbiology}

\section{Stealth bacteria}

\section{Science doi:10.1126/science.1106036 (2004)}

Michinaga Ogawa and colleagues have discovered how Shigella, a bacterium that causes diarrhoea, fever and stomach cramps, evades destruction by host cells. The feat is achieved by means of a camouflage protein that Shigella secretes.

The protein is called IcsB. Bacteria that lack it can still invade cells. But they persist for only about four hours, succumbing to a process in which the host cell wraps them up in the lamellar membrane of the autophagosome, an intracellular trap, where they are eventually destroyed.

How does IcsB enable the bacteria to evade capture for long enough to cause disease? The answer, say Ogawa and colleagues, lies in the bacterial surface protein VirG, a 'red flag' that is targeted by a host protein called Atg5, and which marks bacteria for trapping in the autophagosome. In vitro, Atg5 binds to VirG only when IcsB is absent. It seems that both Atg5 and IcsB can attach to the same binding region in VirG - implying that in host cells IcsB binds to VirG, concealing it from the host protein and allowing Shigella to go about its business undetected. 